\title{
Facial tremor in patients with Parkinson's disease: prevalence, determinants and impacts on disease progression
}

Ruwei Ou, Qianqian Wei, Yanbing Hou, Lingyu Zhang, Kuncheng Liu, Junyu Lin, Zheng Jiang, Bi Zhao, Bei Cao and Huifang Shang ${ }^{*}$

\begin{abstract}
Background: Facial (lip and jaw) tremor (FT) is associated with Parkinson's disease (PD) but few studies have been conducted to explore its clinical profile. We performed this study to investigate the prevalence and clinical correlates of FT in PD, and further to evaluate its effect on disease progression.

Methods: A retrospective, cross-sectional $(n=2224)$ and longitudinal $(n=674)$ study was conducted. The presence of FT was based on $\mathrm{a} \geq 1$ score in the United PD Rating Scale (UPDRS) item 20A. Group comparisons were conducted, followed by a forward binary logistic regression analysis. Inverse probability of treatment weighting (IPTW) based on the propensity score and weighted or unweighted Cox regression models were used to explore the impact of FT on five clinical milestones including death, UPDRS III 11-point increase, Hoehn and Yahr (H\&Y) stage reaching 3, dyskinesia development, and Montreal Cognitive Assessment 3-point decrease.
\end{abstract}

Results: FT was presented in 403 patients (18.1\%), which showed increasing trends with disease duration and H\&Y score. Age $(P<0.001)$, female $(P<0.001)$, disease duration $(P=0.001)$, speech $(P=0.011)$, rigidity $(P=0.026)$, rest tremor on limbs $(P<0.001)$, kinetic tremor on hands $(P<0.001)$, and axial symptoms $(P=0.013)$ were independent factors associated with FT. Both unweighted and weighted Cox regression models indicated that baseline FT and FT as the initial symptom were not associated with the five outcomes.

Conclusions: Our study suggested that FT was not uncommon and provided a deeper insight into the characteristics of FT in PD. The predict value of FT on long-term progronis of PD may need future longer follwe-up study.

Keywords: Parkinson's disease, Facial tremor, Rest tremor, Prevalence, Prognosis

\section{Background}

Parkinson's disease (PD) is a chronic, progressive neurodegenerative disease mainly characterized by rest tremor, rigidity, bradykinesia, and postural and gait disorders [1]. As one of the core motor symptoms, rest tremor is reported in most of the cases (74.4\%) at disease onset [2] and occurs more prevalently in the upper limb rather

\footnotetext{
*Correspondence: hfshang2002@163.com

Department of Neurology, Laboratory of Neurodegenerative Disorders, West China Hospital, Sichuan University, Chengdu 610041, Sichuan, China
}

than lower limb and face [3]. Facial (lip and jaw) tremor (FT) at rest is considered as a relatively uncommon symptom of PD, which can be seen in the early stage of the disease [4]. It is reported that only $1.7 \%$ of patients had FT at disease onset, and its prevalence could be up to $14 \%$ after a mean 9-year disease duration [5]. FT can cause knocking of the teeth, resulting in a vexing sound and social embarrassment, and contribute to the reduced quality of life of patients [6]. 
The exact functional anatomy of rest tremor in PD remains unclear. It is probably related to a combined impairment of the cerebello-dentato-thalamocortical and the basal ganglia-thalamocortical circuits [7]. Rest tremor is often less responsive to dopamine replacement therapy than bradykinesia and rigidity, and its responsiveness to dopaminergic treatment is quite variable among PD patients [8]. In some cases, FT in PD is thought to not respond well to dopaminergic drugs [6]. However, there is also evidence that FT can respond to dopaminergic therapy and has predictive value for clinical PD diagnosis [4]. These controversial results suggest that dopamine deficiency alone may do not determine FT severity, and its response to dopaminergic therapy is likely influenced by multiple factors.

To date, data on the relationships between FT with other PD motor and non-motor symptoms are scarce, and the prevalence of FT is based on a few studies with a small sample size. Although previous analyses of some studies reported that patients with tremor-predominant (TD) subtype have a more benign course [9] and show less severe cognitive deficits [10], a recent study verified that there was no evidence of a benign effect of tremor [11]. So far, it is still unsure the association between FT and PD progression. Thus, we aimed to explore the prevalence and clinical correlates of FT in a large cohort of Chinese PD patients, and then followed up a group of patients at an early stage to further examine whether FT can act as a useful predictor for the progression of PD.

\section{Methods}

\section{Participants}

All procedures of the current study were approved by the Ethics Committee of West China Hospital, Sichuan University. Initially, we enrolled 4332 PD patients from the Department of Neurology, West China Hospital of Sichuan University between March 2009 and July 2019. All participants have provided written informed consent and met the Unified Kingdom PD Society Brain Bank Clinical Diagnostic Criteria for PD [12]. To investigate the prevalence and clinical correlates of FT, we limited our study sample in patients who were assessed at "off" medication $(n=2224)$.

To explore the influence of FT on the disease progression, participants who met the following inclusion criteria were followed up at least once (range 1-10) $(n=$ 725): 1) assessment at "off" medication; 2) disease duration < 3 years; 3) Hoehn and Yahr $(\mathrm{H} \& \mathrm{Y})$ stage < 3; 4) absence of motor complications; and 5) absence of dementia. During the follow-up visit, 21 patients withdraw informed consent and 30 patients lost contact, resulting in the remaining 674 patients who provided information on clinical outcomes were included in the longitudinal data analysis.

\section{Clinical assessments}

Trained neurologists completed standardized assessments of all patients. Demographic and clinical data including sex, age, age at onset, disease duration, education, smoking and drinking history, hypertension, and diabetes mellitus (DM) history, motor complications, and total levodopa equivalent daily dosage (LEDD) were collected. The LEDD was calculated based on a previous systematic review [13]. The Unified PD Rating Scale (UPDRS) part III [14] and H\&Y stage (range 1-5) [15] was used to evaluate the motor symptoms, which was divided into speech, facial expression, tremor at rest, action or postural tremor of hands, rigidity, bradykinesia, and axial symptoms. If possible, all patients were indicated to withdraw medications $>12 \mathrm{~h}$ at follow-up visit ( $n=489,72.6 \%)$. For those without an "off" score, we estimated an "off" medication score by adding the difference value of the study population's mean "off"- and mean "on"-scores to the patient's "on" medication score, as reported by a previous study [16].

The global non-motor symptoms (NMS) was assessed using the Chinese version of the Non-Motor Symptoms Scale (NMSS) [17]. In addition, cognitive function was measured using the Frontal Assessment Battery (FAB; range 1-18) [18] and Montreal Cognitive Assessment (MoCA; range 0-30) [19], with lower scores indicating poor cognition. Depression and anxiety were assessed using the Hamilton Depression Rating Scale (HDRS) (24 items) [20] and the Hamilton Anxiety Rating Scale (HARS), respectively [21]. Freezing of gait was assessed based on scored $\geq 1$ on the Freezing of gait Questionnaire item-13 "Do you feel that your feet get glued to the floor while walking, making a turn, or when trying to initiate walking?" [22]. Falls were determined either with $\mathrm{a} \geq 1$ score on UPDRS-13 (falling unrelated to freezing) or $\mathrm{a} \geqq 3$ score on UPDRS-14 (falling related to freezing).

\section{Determination of FT}

The diagnosis of FT was established by a neurologist trained in movement disorders, and presence or absence explicitly marked on the UPDRS-III score sheet (item 20A: tremor at rest: face, lips, and chin).

\section{Clinical outcomes \\ Survival}

Mortality surveillance was performed mainly throughout the continuing active follow-up of patients and their families. It lasted until July 1, 2020, which was 11 years after our study began (2009), with as many as 14 years of passive follow-up for mortality for patients first diagnosed in 2006. 


\section{Motor decline}

Since a change of 2.5-5.2 points on the UPDRS III score represent a clinically significant difference [23], we thus defined a fast motor progression, based on the mean $3.7 \pm 2.2$ years of follow-up, as an 11-point increased in the UPDRS-III score (mean 3-point per year) and time to such event as the time from the baseline to follow-up visit in which an 11-point increase was first measured.

\section{H\&Y stage}

Time to transfer to H\&Y stage 3 was defined as the time from the baseline to the first follow-up examinations in which the patient scored at least stage 3 .

\section{Dyskinesia}

Time to dyskinesia development was defined as the time from the baseline to the first follow-up examinations in which the patient reported dyskinesia.

\section{Cognitive decline}

Cognitive decline was defined as a 3-point decrease from baseline MoCA score and time to event as the time from the baseline to follow-up examinations in which a 3point decrease was first measured.

\section{Statistical analyses}

Continuous variables were presented as mean \pm standard deviation if normally distributed and as median (interquartile range) if non-normally distributed. Categorical data were shown as number (percentage). To identify the differences in demographic and clinical features at baseline between patients with and without FT, the Student's $T$ test, Wilcoxon rank-sum test, or chi-square test were applied as appropriate.

To explore the potential clinical factors related to FT, a binary logistic regression model was used. The presence or absence of FT was set as the dependent variable and the variables that reported differences between the two groups (selection criterion $P<0.1$ ) or those that were possibly related to FT were chosen as independent variables. The Hosmer and Lemeshow test was implemented to examine the goodness of fit, with $P$ value > 0.05 suggesting high goodness of fit of the model. The tolerance and variance inflation factor (VIF) that were calculated based on a multiple linear regression analysis were used to diagnose the multicollinearity among each independent variable, with tolerance $<0.2$ or VIF $>5$ suggesting the presence of multicollinearity.

Because of significant differences in various baseline characteristics between patients with and without FT (or patients with and without an initial symptom of FT), a propensity score (PS) weighting method was served to balance the differences. The PS model was developed by constructing a logistic regression model in which patients with FT vs. patients without FT (or patients with and without an initial symptom of FT) were regressed on baseline characteristics related to the outcome variables. The estimated PS was achieved as the predicted probability of having FT in each subject. The inverse probability of treatment weighting (IPTW) [24] was then calculated as the inverse of the PS for the patients with FT and as the inverse of (1 - PS) for the patients without FT. To assess bias reduction achieved by the PS weighting, standardized mean differences (SMD) of the confounding covariates that were included for estimating PS were compared between patients with and without FT before and after weighting, with a value of $<$ $10 \%$ indicating between-group balance. All subsequent analyses were weighted by IPTW.

Furthermore, to verify the results from IPTW, a sensitivity analysis was conducted. Multivariate Cox proportional hazards regression models were conducted to calculate hazard ratios (HRs) and 95\% confidence intervals (CIs) of five clinical outcomes across either presence or absence of FT or FT as the initial symptom or not. Two models were conducted. In model 1 , sex, age, and age at onset were adjusted. In model 2 , sex, age, age at onset, BMI, LEDD, UPDRS III score, MoCA score, and NMSS score were adjusted.

Statistical analyses were conducted by $\mathrm{R}$ version 4.0.0 using "Matching", "survey", "reshape2", "survival", and "reportReg" packages. All statistical tests were twotailed, and $P$ values $<0.05$ were regarded as statistically significant.

\section{Results}

\section{Prevalence of FT}

In total, 2224 PD patients (1181 males and 1043 females) were included for retrospective analysis. Among these patients who were assessed UPDRS at "off" medication, 403 patients reported FT (18.1\%). The smooth curves indicated that the prevalence of FT along with limbs tremor at rest or action/postural tremor of hands showed an increasing tendency with increased disease duration and H\&Y stage (Fig. 1).

\section{Comparison between patients with and without FT}

The mean age of the included patients at enrollment was $62.3 \pm 9.9$ years, with a mean onset age of $58.9 \pm 9.9$ years and a mean disease duration of $3.4 \pm 4.3$ years (Table 1 ). The mean UPDRS-III score after exclusion of the FT score was $29.2 \pm 14.2$. Compared with patients without FT, those with FT had significantly lower education, older age, older age of onset, longer disease duration, higher UPDRS III score (exclusion of FT score) as well as higher sub-scores of speech, rest tremor at hands, action or postural tremor, bradykinesia, and axial symptoms, greater $H \& Y$ stage, and lower FAB and MoCA score $(P<0.05)$ (Table 1$)$. 


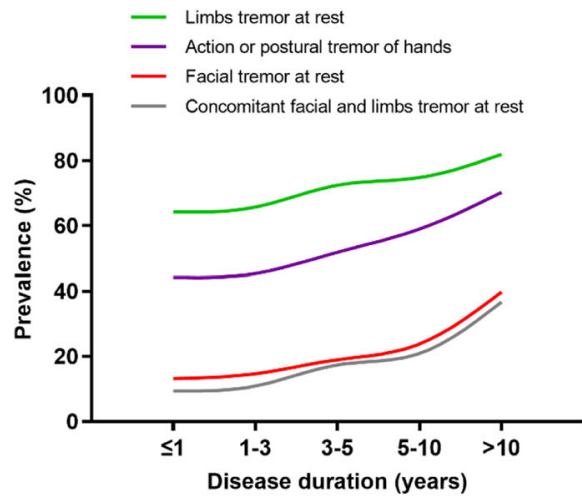

(A)

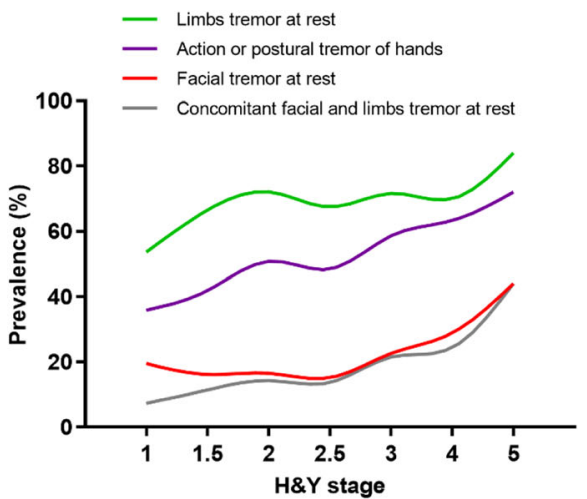

(B)

Fig. 1 Prevalence of tremor in patients with PD with different disease duration and H\&Y stage. The prevalence of each phenotype of tremor including facial tremor at rest, concomitant facial and limbs tremor at rest, limbs tremor at rest as well as action or postural tremor of hands showed an increased tendency with the increased disease duration (a) and H\&Y stage (b)

Table 1 Demographic and clinical features between PD patients with and without FT

\begin{tabular}{|c|c|c|c|c|}
\hline & $\begin{array}{l}\text { Total } \\
(n=2224)\end{array}$ & $\begin{array}{l}\text { With FT } \\
(n=403)\end{array}$ & $\begin{array}{l}\text { Without FT } \\
(n=1821)\end{array}$ & $P$-value \\
\hline Education & $9.5 \pm 4.1$ & $9.0 \pm 4.3$ & $9.6 \pm 4.0$ & $0.016^{*}$ \\
\hline Sex, male & $1181(53.1 \%)$ & $171(42.4 \%)$ & $1010(55.5 \%)$ & $<0.001^{*}$ \\
\hline Age & $62.3 \pm 9.9$ & $65.4 \pm 9.7$ & $61.6 \pm 9.9$ & $<0.001^{*}$ \\
\hline Age at onset & $58.9 \pm 9.9$ & $60.8 \pm 10.1$ & $58.5 \pm 9.9$ & $<0.001^{*}$ \\
\hline Disease duration & $3.4 \pm 3.4$ & $4.5 \pm 4.2$ & $3.1 \pm 3.1$ & $<0.001^{*}$ \\
\hline LEDD & $232.0 \pm 275.7$ & $241.6 \pm 310.0$ & $229.9 \pm 267.6$ & 0.485 \\
\hline UPDRS-III & $29.2 \pm 14.2$ & $32.5 \pm 17.4$ & $28.4 \pm 13.3$ & $<0.001^{*}$ \\
\hline Speech & $0.7 \pm 0.7$ & $0.8 \pm 0.8$ & $0.7 \pm 0.7$ & $<0.001^{*}$ \\
\hline Facial expression & $1.5 \pm 0.8$ & $1.5 \pm 0.9$ & $1.5 \pm 0.8$ & 0.215 \\
\hline Tremor at rest ${ }^{\mathrm{a}}$ & $2.2 \pm 2.4$ & $3.3 \pm 2.9$ & $1.9 \pm 2.1$ & $<0.001^{*}$ \\
\hline Action or postural tremor & $1.6 \pm 1.4$ & $2.2 \pm 1.9$ & $1.4 \pm 1.3$ & $<0.001^{*}$ \\
\hline Rigidity & $8.0 \pm 3.9$ & $8.1 \pm 4.4$ & $8.0 \pm 3.8$ & 0.607 \\
\hline Bradykinesia & $12.3 \pm 6.8$ & $13.2 \pm 7.7$ & $12.0 \pm 6.6$ & $<0.001^{*}$ \\
\hline Axial symptoms & $3.0 \pm 2.7$ & $3.3 \pm 3.1$ & $2.9 \pm 2.5$ & $0.006^{*}$ \\
\hline H\&Y stage & $2.0(0.5)$ & $2.0(0.5)$ & $2.0(0.5)$ & $0.025^{*}$ \\
\hline Freezing of gait & $462(20.8 \%)$ & $95(23.6 \%)$ & $367(20.2 \%)$ & 0.126 \\
\hline Falls & $151(6.8 \%)$ & $32(7.9 \%)$ & $119(6.5 \%)$ & 0.310 \\
\hline Motor fluctuation & $212(9.5 \%)$ & $88(14.7 \%)$ & $340(12.2 \%)$ & 0.087 \\
\hline Dyskinesia & $76(3.4 \%)$ & $33(5.5 \%)$ & $169(6.0 \%)$ & 0.623 \\
\hline$F A B$ & $15.2 \pm 2.7$ & $14.8 \pm 2.8$ & $15.3 \pm 2.7$ & $0.001^{*}$ \\
\hline MoCA & $22.9 \pm 4.8$ & $22.0 \pm 5.1$ & $23.1 \pm 4.7$ & $<0.001^{*}$ \\
\hline HDRS & $9.4 \pm 8.2$ & $9.8 \pm 8.1$ & $9.3 \pm 8.2$ & 0.244 \\
\hline Depression & 727 (32.7\%) & 145 (36.0\%) & $582(32.0 \%)$ & 0.120 \\
\hline HARS & $7.2 \pm 6.6$ & $7.3 \pm 6.7$ & $7.1 \pm 6.6$ & 0.631 \\
\hline Anxiety & $653(29.4 \%)$ & $128(33.8 \%)$ & $525(30.8 \%)$ & 0.242 \\
\hline NMSS & $35.7 \pm 30.5$ & $39.0 \pm 32.9$ & $34.9 \pm 29.9$ & $0.024^{*}$ \\
\hline
\end{tabular}

PD Parkinson's disease, FT Facial tremor, LEDD Levodopa Equivalent Daily Doses, UPDRS Unified PD Rating Scale, H\&Y stage Hoehn and Yahr stage, FAB Frontal Assessment Battery, MoCA Montreal Cognitive Assessment, HDRS Hamilton Depression Rating Scale, HARS Hamilton Anxiety Rating Scale, NMSS Non-Motor Symptoms Scale

${ }^{a}$ The total score was calculated after excluding the score of FT at rest

* Significant difference 


\section{Correlative factors of FT}

Forest map indicated that FT was association with age $(\mathrm{OR}=1.039, \quad 95 \% \mathrm{CI}=1.026-1.053, \quad P<0.001), \quad$ females $(\mathrm{OR}=1.896, \quad 95 \% \mathrm{CI}=1.492-2.409, \quad P<0.001), \quad$ disease duration $(\mathrm{OR}=1.067,95 \% \mathrm{CI}=1.029-1.107, P=0.001)$, speech $(\mathrm{OR}=1.297,95 \% \mathrm{CI}=1.062-1.583, P=0.011)$, rigidity $\quad(\mathrm{OR}=0.958, \quad 95 \% \mathrm{CI}=0.922-0.995, \quad P=0.026)$, limbs tremor at rest $(\mathrm{OR}=1.165,95 \% \mathrm{CI}=1.108-1.225$, $P<0.001)$, action or postural tremor of hands $(\mathrm{OR}=$ $1.246,95 \% \mathrm{CI}=1.151-1.350, P<0.001)$, and axial symptoms $\quad(\mathrm{OR}=0.926, \quad 95 \% \mathrm{CI}=0871-0.984, \quad P=0.013)$ (Fig. 2). The tolerance of each independent variable was more than 0.2 and all the VIF were less than 5 (Supplementary Table 1), suggesting there was no multicollinearity presented in the model. The Hosmer and Lemeshow test showed that the goodness of fit of the model was superior $(P=0.686)$. The percentage accuracy in the classification of the model was $81.9 \%$.

\section{Effects of FT on clinical outcomes}

Of the 674 participants who completed the follow-up visits, 65 patients died (9.6\%), with a mean time to death or censoring from baseline of $5.7 \pm 2.3$ years; $203(30.1 \%)$ reported a decrease of at least 11 points in UPDRS III score after $3.7 \pm 2.2$ years of follow up; 119 (17.7\%) patients reached at least H\&Y stage 3 after a mean disease duration of $4.9 \pm 2.0$ years; 161 (23.9\%) patients reported a decrease of at least 3 points in MoCA score after a mean $3.6 \pm 2.1$ years of follow-up; and 94 (13.9\%) reported new occurrence of dyskinesia after a mean $5.1 \pm 2.1$ years of follow up. The mean change in the UPDRS III score from baseline to follow-up visit was $-7.9 \pm 10.8$ points, while the mean change in the MoCA score was $-0.7 \pm 3.1$ points, after excluding the patients who had died.

IPTW was formed by those with complete data on clinical outcomes. A total of 18 covariates at baseline including sex, age, age of onset, disease duration, drinking, smoking, hypertension, DM, BMI, education, LEDD, UPDRS III score, H\&Y stage, MoCA score, FAB score, HDRS score, HARS score, and NMSS score were included for estimating the propensity score (Fig. 3). Compared to pre-weighting, the SMD values of each variable at baseline were reduced after-weighting. All the SMD values were $<10 \%$, suggesting there was a betweengroup balance on baseline characteristics after weighting (Fig. 3 and Supplementary Table 2).

The univariate Cox model indicated that baseline FT and FT as the initial symptom in the unweighted PD population were associated with survival $(P=0.006$ and $P<0.001$, respectively), but this association disappeared after using the weighted method by IPTW $(P=0.166$ and $P=0.342$, respectively) (Table 2). Baseline FT and FT as the initial symptom were also not associated with time to UPDRS-III 11-point increase, time to convert to H\&Y stage 3, time to dyskinesia, and time to MoCA 3point decrease both before and after matching $(P>0.05)$ (Table 2). In the sensitivity analysis, the multivariate Cox regression model suggested that baseline FT and FT as the initial symptom were not associated with the five clinical outcomes after adjusting confounding factors $(P>0.05$, both in model 1 and model 2$)$ (Table 3).

\section{Discussion}

We explored the prevalence and clinical correlates of FT in a large cohort of patients with PD. In a sample of

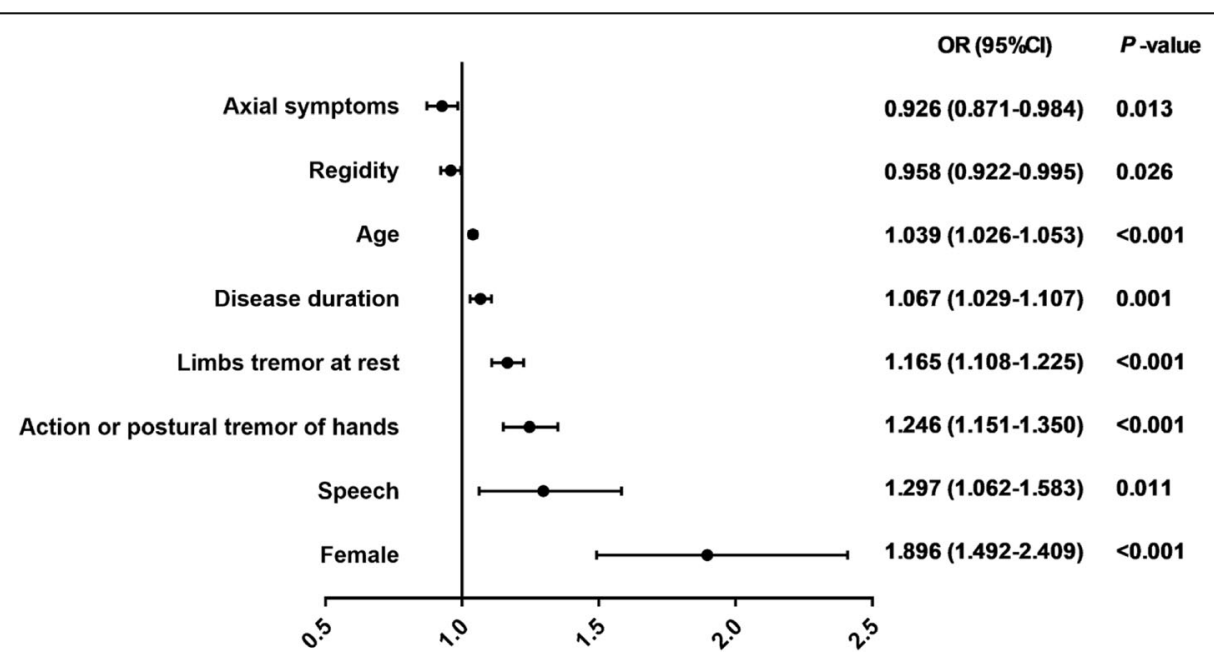

Fig. 2 Correlative factors of facial tremor at rest in patients with PD. Forest map showed correlative factors of facial tremor at rest in a group of PD patients who were assessed at "off" medication state. In this model, the following controlled covariates were non-significant: education, facial expression score, bradykinesia score, FAB score, MoCA score, and NMSS score. All OR, 95\%Cl, and P-values were calculated from a forward binary logistic regression analysis 


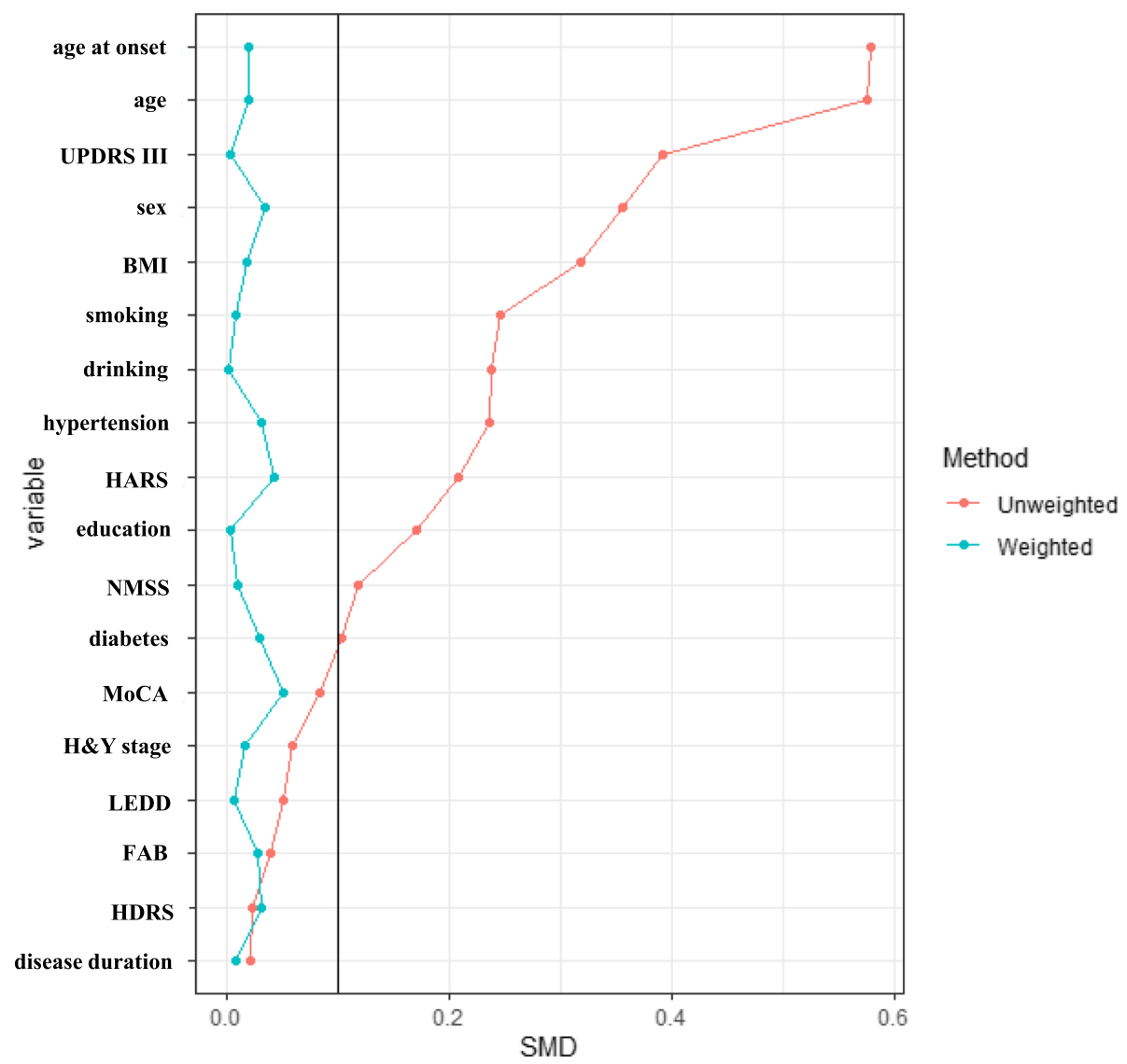

Fig. 3 SMD in baseline covariates between patients with and without FT before and after IPTW. SMD of baseline confounding covariates that were included for estimating the propensity scores before and after weighting. All the values of SMD were $<10 \%$ after IPTW weighting, which indicated there was a between-group balance after weighting. UPDRS III: Unified Parkinson's disease Rating Scale part III. H\&Y stage: Hoehn and Yahr stage. BMl: Body Mass Index. FAB: Frontal Assessment Battery. MoCA: Montreal Cognitive Assessment. HDRS: Hamilton Depression Rating Scale. HARS: Hamilton Anxiety Rating Scale. NMSS: Non-Motor Symptoms Scale. LEDD: levodopa equivalent daily dosage. SMD: Standardized Mean Differences. FT: facial tremor. IPTW: inverse probability of treatment weighting

Table 2 Univariate Cox model for exploring the association between FT and clinical outcomes in PD before and after weighting

\begin{tabular}{|c|c|c|c|c|}
\hline & \multicolumn{2}{|c|}{ Unweighted sample } & \multicolumn{2}{|c|}{ Weighted sample } \\
\hline & $\mathrm{HR}(95 \% \mathrm{Cl})$ & $P$-value & $\mathrm{HR}(95 \% \mathrm{Cl})$ & $P$-value \\
\hline \multicolumn{5}{|l|}{ Presence of FT at baseline } \\
\hline Time to death & $0.41(0.22-0.80)$ & $0.006^{*}$ & $0.62(0.31-1.22)$ & 0.166 \\
\hline Time to UPDRS-III 11-point increase & $0.77(0.43-1.30)$ & 0.339 & $0.98(0.54-1.76)$ & 0.934 \\
\hline Time to conversion to $\mathrm{H} \& \mathrm{Y}$ stage $\geq 3$ & $0.98(0.50-1.78)$ & 0.938 & $0.66(0.33-1.30)$ & 0.224 \\
\hline Time to dyskinesia & $0.41(0.14-0.95)$ & 0.061 & $0.44(0.17-1.17)$ & 0.101 \\
\hline Time to MoCA 3-point decrease & $0.93(0.51-1.61)$ & 0.793 & $0.67(0.36-1.23)$ & 0.198 \\
\hline \multicolumn{5}{|l|}{ FT as initial symptom } \\
\hline Time to death & $2.82(1.55-5.11)$ & $<0.001^{*}$ & $1.75(0.55-5.54)$ & 0.342 \\
\hline Time to UPDRS-III 11-point increase & $0.97(0.60-1.55)$ & 0.889 & $0.74(0.24-2.31)$ & 0.606 \\
\hline Time to conversion to $\mathrm{H} \& \mathrm{Y}$ stage $\geq 3$ & $1.24(0.69-2.12)$ & 0.467 & $0.34(0.08-1.56)$ & 0.167 \\
\hline Time to dyskinesia & $0.51(0.21-1.27)$ & 0.149 & $0.81(0.18-3.70)$ & 0.790 \\
\hline Time to MoCA 3-point decrease & $1.04(0.63-1.72)$ & 0.885 & $0.45(0.13-1.63)$ & 0.226 \\
\hline
\end{tabular}

FT Facial tremor, PD Parkinson's disease, UPDRS Unified PD Rating Scale, MoCA Montreal Cognitive Assessment * Significant difference 
Table 3 Multivariate Cox regression model for exploring the association between FT and clinical outcomes in patients with PD

\begin{tabular}{|c|c|c|c|c|}
\hline & \multicolumn{2}{|l|}{ Model 1} & \multicolumn{2}{|l|}{ Model 2} \\
\hline & HR $(95 \% \mathrm{Cl})$ & $P$-value & $\mathrm{HR}(95 \% \mathrm{Cl})$ & $P$-value \\
\hline \multicolumn{5}{|l|}{ Presence of FT at baseline } \\
\hline Time to death & $1.65(0.87-3.12)$ & 0.122 & $1.50(0.78-2.90)$ & 0.230 \\
\hline Time to UPDRS-III 11-point increase & $1.23(0.75-2.01)$ & 0.421 & $1.21(0.73-2.00)$ & 0.460 \\
\hline Time to conversion to $\mathrm{H} \& \mathrm{Y}$ stage $\geq 3$ & $0.95(0.52-1.74)$ & 0.876 & $0.93(0.51-1.72)$ & 0.822 \\
\hline Time to dyskinesia & $0.64(0.25-1.61)$ & 0.340 & $0.57(0.23-1.45)$ & 0.241 \\
\hline Time to MoCA 3-point decrease & $0.97(0.57-1.64)$ & 0.903 & $0.97(0.57-1.65)$ & 0.907 \\
\hline \multicolumn{5}{|l|}{ FT as initial symptom } \\
\hline Time to death & $2.46(0.90-6.76)$ & 0.080 & $2.56(0.91-7.23)$ & 0.075 \\
\hline Time to UPDRS-III 11-point increase & $0.91(0.33-2.52)$ & 0.861 & $0.86(0.31-2.42)$ & 0.777 \\
\hline Time to conversion to H\&Y stage $\geq 3$ & $0.47(0.11-1.96)$ & 0.300 & $0.49(0.12-2.06)$ & 0.332 \\
\hline Time to dyskinesia & $1.07(0.25-4.47)$ & 0.931 & $1.09(0.26-4.63)$ & 0.906 \\
\hline Time to MoCA 3-point decrease & $0.54(0.17-1.74)$ & 0.301 & $0.50(1.16-1.63)$ & 0.253 \\
\hline
\end{tabular}

FT Facial tremor, PD Parkinson's disease, UPDRS Unified PD Rating Scale, MoCA Montreal Cognitive Assessment Model 1, adjusted for sex, age, and age at onset

Model 2, adjusted for sex, age, age at onset, BMI, LEDD, UPDRS III score, MoCA score, and NMSS score

patients who were assessed at "off" medication, FT was present in $18.1 \%$ of cases and independently associated with females, older age, and longer disease duration in addition to the core motor symptoms. Furthermore, we also assessed the prognostic value of FT on disease progression of PD and found that both the presence of FT and FT as the initial symptom were not associated with survival, dyskinesia, as well as motor and cognitive decline.

The prevalence of FT reported by previous studies $[5,25]$ was lower than our findings. In a previous study that included patients with disease duration $<2$ years, the authors revealed that only $1.5 \%(4 / 263)$ patients had FT [25]. Another study included 50 patients with longer disease duration (mean $9.0 \pm 6.6$ years) and found $14 \%$ of patients had FT [5]. The strength of the current study is that we recruited a large sample population with all H\&Y stages. Moreover, our study disclosed that the prevalence of FT was increased with advanced disease duration and H\&Y stage, which can explain the complexity of symptomatology of the disease in the advanced stage and is likely to increase the difficulty to manage this symptom over time. Our findings also indirectly confirm that the evidence proposed that the tremor in PD may spread to other sites with the progression of the disease [2].

In the current study, we found that FT was associated with older age and longer disease duration. Although the likelihood of developing FT increases over time in patients with $\mathrm{PD}$, the age and duration of the disease itself cannot be totally responsible, because not all patients with PD will eventually develop tremors. Furthermore, it is interesting that we found that female patients showed more susceptibility to experience with FT, which should be further pathologically verified since men are reported at a higher risk for PD and present with a faster deterioration of motor and non-motor functions [26].

In looking for the clinical correlates of FT with other motor symptoms, we found that both rest and kinetic tremors on limbs were positively correlated with FT. Such association suggests tremors occurred in PD no matter in limbs or facial areas may share some common pathophysiological mechanisms. It is noteworthy that different body parts may have similar tremor frequencies in PD, but are generally not the same and are not phaselocked [27]. This suggests that each body part has a separate tremor generator. The ability to stay separation may be due to the somatotopic segregation of basal ganglia loops [27]. Furthermore, it must be emphasized that tremor in PD may have multifaceted phenomenology and, probably, pathophysiology [27].

The association between FT and speech disorders indicates that the neural network among these symptoms may connect. One explanation for such a phenomenon is that subthalamic stimulation can cause a significant deterioration of speech or improve loudness of speech while relieving tremor [28]. In addition, the negative association between rigidity and FT is probably due to the fact that patients with rest tremor usually presented with slighter rigidity compared to those who do not exhibit rest tremor [25]. The neurophysiological similarities between tremors and the cogwheel phenomenon usually associated with rigidity can explain such association [29]. Moreover, the greater improvement of tremor and rigidity by deep brain stimulation [30] is another possible explanation. However, lack of relationship between FT and bradykinesia, another cardinal motor sign of PD that is strongly responsive to dopaminergic drugs, and 
relates to the dopaminergic cell loss in the substantia nigra [31], supports a contribution of non-dopaminergic mechanisms involving in FT. Although the response to levodopa of FT was not assessed in the current study, lack of association between FT and LEDD supports the above hypothesis.

In the current study, we found that both baseline FT and FT as the initial symptom were not associated with motor and non-motor progression, suggesting that FT is not a prognostic indicator for monitoring the progression of PD. Based on the ratio of tremor and nontremor score, patients with PD were usually classified as TD and posture instability gait disorder (PIGD) subtypes. It is reported that patients with the TD subtype have a more benign course compared to those with the non-TD subtype [9]. Based on our finding that FT does not contribute to the disease progression, it is better not to be considered FT as a contributor in the TD subtype when calculating the ratio of tremor and PIGD to classify the PD subtypes.

Actually, a recent study [11] verified the severity of PIGD could be more appropriate as a clinical biomarker for the disease progression and the authors also did not support the use of the TD subtype as a prognostic trait in PD. This is because several longitudinal studies found that patients may switch their initial motor subtype from TD to PIGD, a transition that seems unidirectional $[31,32]$. Since patients with the PIGD subtype show faster progression than those with the TD subtype, and subtype classification of patients is based on a ratio score of tremor/non-tremor symptoms, the switch from the TD subtype to the PIGD subtype is basically driven by increased severity of PIGD. However, as we stated earlier, each body part has a separate tremor generator, so our study suggests that a more complicated pathophysiology may involve in the development of FT compared to rest tremor at hands. Therefore, the prognostic value of each type of tremor should not be based on the ratio method. Instead, the effect of tremor and PIGD severity should be individually evaluated.

Some limitations should be considered. First, we focused on a group of patients who were assessed at "off" medication and therefore selection bias cannot be ruled out. Second, the relatively short observation of FT on clinical outcomes is not enough to conclude the influence of FT on long-term outcomes such as death. Third, the assessment of FT was based on UPDRS, a scale that does not distinguish between lips and chin tremor, resulting in these variables could not be used for stratification.

\section{Conclusions}

FT is not rare in patients with PD, which can be affected by sex, age as well as disease duration and motor severity. Baseline FT and FT as the initial symptom are not associated with the disease progression of $\mathrm{PD}$. The predictive value of FT on long-term progronis of $\mathrm{PD}$ may need to clarify by future longer follow-up studies.

\section{Supplementary Information}

The online version contains supplementary material available at https://doi. org/10.1186/s12883-021-02105-y

Additional file 1: Supplementary Table 1. Diagnosis of

multicollinearity for each candidate variate. Supplementary Table 2.

SMD of baseline covariates before and after IPTW between groups

\section{Acknowledgments}

The authors thank the patients and their families for their participation in the study.

\section{Authors' contributions}

ORW contributed with conception, organization and execution, data collection and statistical analysis, and drafting the manuscript. WQQ, HYB, ZLY, LKC, LJY, JZ, ZB, and CB contributed with execution, data collection and statistical analysis. SHF contributed with conception and organization, manuscript review and critique, and resposible for overall content as the guarantor. All arthors read and approved the final manuscript.

\section{Funding}

This study was supported by the 1.3.5 project for disciplines of excellence, West China Hospital, Sichuan University (ZYJC18038), and the National Clinical Research Center for Geriatrics, West China Hospital, Sichuan University (Z2018B08)

Availability of data and materials

All data generated or analysed during this study are available by reasonable requiry. (Please contact: Huifang Shang, email: hfshang2002@126.com).

\section{Ethics approval and consent to participate}

This study was conducted in agreement with the Ethics Committee of Sichuan University West China Hospital. All participants had provided written informed consent.

\section{Consent for publication}

None.

\section{Competing interests}

The authors declare that they have no conflict of interest.

Received: 7 October 2020 Accepted: 9 February 2021

Published online: 23 February 2021

References

1. Schapira AHV, Chaudhuri KR, Jenner P. Erratum: Non-motor features of Parkinson disease. Nat Rev Neurosci. 2017;18(8):509. https://doi.org/10.1038/ nrn.2017.91

2. Gigante AF, Pellicciari R, Iliceto G, Liuzzi D, Mancino PV, Custodero GE, et al. Rest tremor in Parkinson's disease: body distribution and time of appearance. J Neurol Sci. 2017;375:215-9. https://doi.org/10.1016/j.jns.201 6.12.057.

3. Jankovic J. Parkinson's disease: clinical features and diagnosis. J Neurol Neurosurg Psychiatry. 2008;79(4):368-76. https://doi.org/10.1136/jnnp.2 007.131045

4. Rossi M, Wilken M, Morisset P, Fariña S, Cerquetti D, Merello M. Facial tremors in patients with and without parkinsonism. Neurol Sci. 2016;37(12): 1999-2002. https://doi.org/10.1007/s10072-016-2683-x.

5. Sternberg EJ, Alcalay RN, Levy OA, Louis ED. Postural and intention tremors: a detailed clinical study of essential tremor vs. Parkinson's Disease. Front Neurol. 2013;4:51. https://doi.org/10.3389/fneur.2013.00051.

6. Schneider SA, Edwards MJ, Cordivari C, Macleod WN, Bhatia KP. Botulinum toxin a may be efficacious as treatment for jaw tremor in Parkinson's disease. Movement Disord. 2006;21(10):1722-4. https://doi.org/10.1002/ mds.21019. 
7. Boecker $H$, Brooks DJ. Resting tremor in Parkinson disease: is the pallidum to blame? Ann Neurol. 2011;69(2):229-31. https:/doi.org/10.1002/ana.22358.

8. Fishman PS. Paradoxical aspects of parkinsonian tremor. Mov Disord. 2008; 23(2):168-73. https://doi.org/10.1002/mds.21736.

9. Jankovic J, McDermott M, Carter J, Gauthier S, Goetz C, Golbe L, et al. Variable expression of Parkinson's disease: a base-line analysis of the DATA TOP cohort. The Parkinson Study Group. Neurology. 1990;40(10):1529-34. https://doi.org/10.1212/wnl.40.10.1529.

10. Wojtala J, Heber IA, Neuser P, Heller J, Kalbe E, Rehberg SP, et al. Cognitive decline in Parkinson's disease: the impact of the motor phenotype on cognition. J Neurol Neurosurg Psychiatry. 2019;90(2):171-9. https://doi.org/1 0.1136/jnnp-2018-319008.

11. van der Heeden JF, Marinus J, Martinez-Martin P, Rodriguez-Blazquez C, Geraedts VJ, van Hilten JJ. Postural instability and gait are associated with severity and prognosis of Parkinson disease. Neurology. 2016;86(24):224350. https://doi.org/10.1212/wnl.0000000000002768.

12. Hughes AJ, Daniel SE, Kilford L, Lees AJ. Accuracy of clinical diagnosis of idiopathic Parkinson's disease: a clinico-pathological study of 100 cases. J Neurol Neurosurg Psychiatry. 1992;55(3):181-4. https://doi.org/10.1136/jnnp.55.3.181.

13. Tomlinson CL, Stowe R, Patel S, Rick C, Gray R, Clarke CE. Systematic review of levodopa dose equivalency reporting in Parkinson's disease. Movement Disord. 2010;25(15):2649-53. https://doi.org/10.1002/mds.23429.

14. Goetz CG, Fahn S, Martinez-Martin P, Poewe W, Sampaio C, Stebbins GT, et al. Movement Disorder Society-sponsored revision of the unified Parkinson's disease rating scale (MDS-UPDRS): process, format, and clinimetric testing plan. Movement Disord. 2007;22(1):41-7. https://doi.org/1 $0.1002 / m d s .21198$

15. Hoehn MM, Yahr MD. Parkinsonism: onset, progression and mortality. Neurology. 1967;17(5):427-42.

16. Ritz B, Rhodes SL, Bordelon Y, Bronstein J. Alpha-Synuclein genetic variants predict faster motor symptom progression in idiopathic Parkinson disease. PLoS One. 2012;7(5):e36199. https://doi.org/10.1371/journal.pone.0036199.

17. Wang G, Hong Z, Cheng Q, Xiao Q, Wang Y, Zhang J, et al. Validation of the Chinese non-motor symptoms scale for Parkinson's disease: results from a Chinese pilot study. Clin Neurol Neurosurg. 2009;111(6):523-6. https://doi. org/10.1016/j.clineuro.2009.02.005.

18. Rothlind JC, Brandt J. A brief assessment of frontal and subcortical functions in dementia. J Neuropsychiatry Clin Neurosc. 1993;5(1):73-7. https://doi. org/10.1176/jnp.5.1.73.

19. Nasreddine ZS, Phillips NA, Bedirian V, Charbonneau S, Whitehead V, Collin I, et al. The Montreal cognitive assessment, MoCA: a brief screening tool for mild cognitive impairment. J Am Geriatr Soc. 2005;53(4):695-9. https://doi. org/10.1111/j.1532-5415.2005.53221.x.

20. Hamilton M. Development of a rating scale for primary depressive illness. $\mathrm{Br}$ J Soc Clin Psychol. 1967;6(4):278-96. https://doi.org/10.1111/j.2044-8260.1 967.tb00530.x.

21. Clark DB, Donovan JE. Reliability and validity of the Hamilton anxiety rating scale in an adolescent sample. J Am Acad Child Adolesc Psychiatry. 1994; 33(3):354-60. https://doi.org/10.1097/00004583-199403000-00009.

22. Giladi N, Tal J, Azulay T, Rascol O, Brooks DJ, Melamed E, et al. Validation of the freezing of gait questionnaire in patients with Parkinson's disease. Mov Disord. 2009;24(5):655-61. https://doi.org/10.1002/mds.21745.

23. Shulman LM, Gruber-Baldini AL, Anderson KE, Fishman PS, Reich SG, Weiner WJ. The clinically important difference on the unified Parkinson's disease rating scale. Arch Neurol. 2010;67(1):64-70. https://doi.org/10.1001/a rchneurol.2009.295.

24. Cole SR, Hernan MA. Constructing inverse probability weights for marginal structural models. Am J Epidemiol. 2008;168(6):656-64. https://doi.org/10.1 093/aje/kwn164.

25. Pasquini J, Ceravolo R, Qamhawi Z, Lee JY, Deuschl G, Brooks DJ, et al. Progression of tremor in early stages of Parkinson's disease: a clinical and neuroimaging study. Brain. 2018;141(3):811-21. https://doi.org/10.1093/bra in/awx376.

26. Gillies GE, Pienaar IS, Vohra S, Qamhawi Z. Sex differences in Parkinson's disease. Front Neuroendocrinol. 2014;35(3):370-84. https://doi.org/10.1016/j. yfrne.2014.02.002.

27. Hallett M. Parkinson's disease tremor: pathophysiology. Parkinsonism Relat Disord. 2012;18(Suppl 1):S85-6. https://doi.org/10.1016/S1353-8020(11)70027-X.

28. Strupp M. Parkinson's disease: news on tremor, subthalamic stimulation and impulse control disorders. J Neurol. 2011;258(2):340-2. https://doi.org/10.1 007/s00415-011-5916-1.
29. Findley LJ, Gresty MA, Halmagyi GM. Tremor, the cogwheel phenomenon and clonus in Parkinson's disease. J Neurol Neurosurg Psychiatry. 1981;44(6): 534-46. https://doi.org/10.1136/jnnp.44.6.534.

30. Benabid AL, Chabardes S, Mitrofanis J, Pollak P. Deep brain stimulation of the subthalamic nucleus for the treatment of Parkinson's disease. Lancet Neurol. 2009;8(1):67-81. https://doi.org/10.1016/s1474-4422(08)70291-6.

31. Burn DJ, Rowan EN, Allan LM, Molloy S, O'Brien JT, McKeith IG. Motor subtype and cognitive decline in Parkinson's disease, Parkinson's disease with dementia, and dementia with Lewy bodies. J Neurol Neurosurg Psychiatry. 2006;77(5):585-9. https://doi.org/10.1136/jnnp.2005.081711.

32. Alves G, Larsen JP, Emre M, Wentzel-Larsen T, Aarsland D. Changes in motor subtype and risk for incident dementia in Parkinson's disease. Movement Disord. 2006;21(8):1123-30. https://doi.org/10.1002/mds.20897.

\section{Publisher's Note}

Springer Nature remains neutral with regard to jurisdictional claims in published maps and institutional affiliations.
Ready to submit your research? Choose BMC and benefit from:

- fast, convenient online submission

- thorough peer review by experienced researchers in your field

- rapid publication on acceptance

- support for research data, including large and complex data types

- gold Open Access which fosters wider collaboration and increased citations

- maximum visibility for your research: over $100 \mathrm{M}$ website views per year

At BMC, research is always in progress.

Learn more biomedcentral.com/submissions 